\title{
Percepción de los Estudiantes sobre el Aprendizaje Significativo y Estrategias de Enseñanza en el Blended Learning
}

\section{Perception of Students on Meaningful Learning and Teaching Strategies in Blended Learning}

\author{
María del Rocío Carranza Alcántar * \\ Juan Francisco Caldera Montes \\ Universidad de Guadalajara, México
}

\begin{abstract}
Uno de los retos más importantes para la investigación educativa en los últimos años ha sido conocer de qué manera las TIC y las estrategias de enseñanza utilizadas por los docentes impactan en el aprendizaje significativo de los estudiantes, pues a pesar de que se están realizando constantes acciones pedagógicas que permitan combinar las TIC con la formación tradicional, modalidad educativa llamada blended learning, aún no se cuentan con resultados concretos que permitan demostrar el éxito que tienen este tipo de acciones. El objetivo es reconocer la percepción que tienen los estudiantes del aprendizaje significativo que alcanzan y las estrategias de enseñanza que utilizan sus profesores en este tipo de modalidades. Para el cumplimiento de tal objetivo, se diseñó y validó la denominado Escala de Percepción de Aprendizaje Significativo en el Blended Learning (E.P.A.S.B.L.), la cual mostró adecuadas propiedades psicométricas, lo que permite considerarse como válido y confiable. La muestra estuvo integrada por 110 alumnos de la carrera de abogado de una institución pública. Los resultados muestran que los participantes tienen la percepción de que su nivel de aprendizaje significativo combinado con las estrategias de enseñanza que utilizan sus profesores alcanza niveles medios, existiendo diferencias significativas conforme a la edad de los mismos, lo cual sugiere poner mayor énfasis en el tipo de estrategias utilizadas en el blended learning.
\end{abstract}

Descriptores: Percepción; Estudiantes; Aprendizaje; Método de enseñanza; Tecnología de la información.

One of the most important challenges for educational research in recent years has been to know how ICT and the teaching strategies used by teachers impact on students' meaningful learning, because although there are constant pedagogical actions that allow combining ICT with traditional training, educational modality called blended learning, there are still no concrete results to demonstrate the success that these types of actions have. The aim is to recognize the perception that students have of the significant learning they achieve and the teaching strategies used by their teachers in this type of modality. To achieve this aim, the Significant Learning Perception Scale in Blended Learning (S.L.P.S.B.L.) was designed and validated, which showed adequate psychometric properties, which allows it to be considered as valid and reliable. The sample was integrated by 110 students of the career of law of a public institution. The results show that participants have the perception that their level of meaningful learning combined with the teaching strategies used by their teachers reach average levels, there being significant differences according to their age, which suggests placing greater emphasis on the type of strategies used in the blended learning.

Keywords: Perception; Students; Learning; Teaching methods; Blended learning; Information technology.

*Contacto: mcarranza@cualtos.udg.mx

ISSN: 1696-4713

www.rinace.net/reice/

revistas.uam.es/reice
Recibido: $\quad 21$ de agosto 2017

$1^{a}$ Evaluación: 1 de noviembre 2017

$2^{\text {a }}$ Evaluación: 9 de diciembre 2017

Aceptado: 15 de diciembre 2017 


\section{Introducción}

En los últimos años numerosas instituciones de educación superior han experimentado cambios y realizando iniciativas que, con base en las Tecnologías de la Información y la Comunicación (TIC), pretenden promover que los estudiantes desarrollen conocimientos científicos rigurosos, integrados y aplicados en su desempeño profesional, además de fomentar en ellos la capacidad de ser aprendices autónomos y autogestivos, así como participar en la consolidación de ciudadanos críticos, reflexivos, creativos y responsables (Monereo, 2013; UNESCO, 2014).

Lo anterior pone de manifiesto, lo que han señalado González, Padilla y Rincón (2012) y más recientemente Aguaded y Cabero Almenara (2014) en relación a que uno de los retos que se están presentando en la actualidad, principalmente en el ámbito de la formación digital, es realizar acciones pedagógicas que combinen la formación tradicional con la no presencial o en línea, denominada blended learning o también conocida como modalidad mixta, las cuales se desarrollan con el soporte de plataformas y redes, abriendo de esta manera nuevos horizontes y provocando que el aprendizaje sea cada vez más universal, puesto que rompe los límites del tiempo y del espacio.

Tal afirmación encuentra sustento en lo señalado por Islas-Torres (2015), quien sostiene que las modalidades no convencionales han empezado a incrementarse en los centros educativos como una respuesta a las necesidades formativas, esto es, como un agregado de tecnologías en el aula, combinando la virtualidad con la presencialidad; de tal manera que resulta necesario trabajar en los cambios educativos que esta situación conlleva, buscando además conocer el impacto que estas modalidades tienen tanto en la enseñanza y primordialmente en el aprendizaje.

Ante dichas condiciones conviene investigar sobre el estado que guardan las modalidades educativas que son mediadas por las nuevas tecnologías, de manera particular sobre la relación que han tenido las mismas en el aprendizaje de los estudiantes (Bos, Ganimian y Vegas, 2015), especialmente sobre las denominadas estrategias de enseñanza ya que hoy en día se ha reconocido que la planificación adecuada de éstas representa una de las prioridades y metas para el desarrollo de la educación vía las TIC (OEI, 2015). De igual manera se ha hablado de la importancia de conocer las percepciones o concepciones de los estudiantes, ya que a partir de esto se podrá mejorar la práctica educativa y por lo tanto lograr un aprendizaje integral (Hidalgo y Murillo, 2017).

Para estos casos, Díaz Barriga (2005) señala que la función del profesor en la actualidad debe consistir en generar oportunidades de aprendizaje, mediante las cuales el alumno pueda cuestionarse qué es lo que ya conoce acerca del tema a desarrollar, buscar nueva información, y trabajar en colaboración con otros para resolver problemas y tomar decisiones; es decir, el docente requiere de un profundo conocimiento no solo del contenido de su asignatura, sino de estrategias de enseñanza que apoyen el aprendizaje (Marcelo et al., 2016). De este modo, el docente es responsable de guiar a sus alumnos facilitándoles el acceso a recursos que les permitan explorar y elaborar nuevos conocimientos, lo cual ayuda a fomentar el desarrollo de habilidades y destrezas.

En este sentido se han realizado diversos estudios relacionados con el proceso de enseñanza aprendizaje en las modalidades digitales y/o mixtas, como es el caso de Bartolomé (2004), Osbaldo (2010), Laster (2005), Garnham y Kaleta (2002), Ball y Pelco (2006) y Peña (2010) y recientemente por García-Valcárcel y Tejedor Tejedor (2017), en 
las que se habla de algunos casos de éxito al utilizar las TIC como apoyo al aprendizaje; sin embargo, se considera que falta profundizar sobre la temática.

Con base en estos preceptos es que se pensó conveniente estudiar desde la perspectiva de los estudiantes universitarios la percepción que éstos tienen sobre el nivel de aprendizaje significativo que alcanzan en una modalidad educativa que combina la presencialidad con la virtualidad, es decir, un modelo mixto de enseñanza aprendizaje y su relación con las estrategias de enseñanza que utilizan sus profesores. Ante tal contexto este estudio pretende realizar dicha investigación, así como esclarecer los constructos teóricos que la sustentan, y que continuación se desarrollan.

\section{Fundamentación teórica}

En especial y para esta investigación se ha señalado que el aprendizaje significativo refiere la siguiente idea: la integración de los nuevos conocimientos en la estructura cognitiva de quien aprende supone ciertas condiciones, a saber, la presencia de ideas previas para relacionar el conocimiento previo con el nuevo y, sobre todo, que sean tomadas en cuenta por el profesor-mediador; la significación potencial del material, es decir, un material estructurado lógicamente y una actitud activa, tanto del estudiante para aprender como del mediador para propiciar la construcción de los conocimientos. Por lo tanto, se puede expresar que resulta imprescindible pensar las cosas para conocerlas, ya que su representación implica nuevas conexiones entre ellas y el hombre, las cuales hacen sentir su presencia en la medida en que se establecen otras relaciones, más allá de la realidad inmediata (Ausubel, 2002).

En cuanto al grado máximo de significatividad posible de un aprendizaje, en teoría no existen límites, ya que siempre es posible, en principio, añadir nuevos significados a los ya construidos o establecer nuevas y más complejas relaciones entre ellos. De tal manera que el aprendizaje significativo no es una cuestión de todo o nada, sino de grado. No cabe diseñar una actividad de evaluación con el propósito de discernir si el aprendizaje que han realizado los alumnos es o no significativo, lo que procede es detectar el grado de significatividad del aprendizaje realizado, utilizando para ello actividades y tareas susceptibles de ser abordadas o resueltas a partir de diferentes grados de significatividad de los contenidos implicados en su desarrollo o resolución (Coll, 2007, 2014).

Por lo tanto, y siguiendo en este tenor, para que el aprendizaje significativo pueda concretarse se requiere de una serie de acciones y/o cogniciones en cada uno de los estudiantes. Dichas acciones pueden denominarse dimensiones del aprendizaje significativo, mismas que fueron utilizadas como base para diseñar el instrumento que en esta investigación se presenta y que se sintetizan a continuación.

\subsection{Dimensiones del aprendizaje significativo}

Primera dimensión: La motivación

La motivación se traduce en un compromiso real con el proceso de aprendizaje por parte del estudiante (Zarzar, 2000). Es decir, el mayor o menor grado de significatividad del aprendizaje dependerá en gran parte de la fuerza de esa tendencia a aprender significativamente.

Siguiendo este contexto Riveros (2009) y Espinoza (2017) señalan que se requiere de una predisposición para aprender significativamente y para efectuar el esfuerzo mental que eso 
supone. De aquí la importancia de que la función principal del profesor sea que sus alumnos aprendan de manera significativa. En este sentido se retoma lo manifestado por Pérez Gómez (2008) y Pérez Sánchez y Beltrán Llera (2014) respecto a que la motivación es el motor del aprendizaje que se encuentra influida por las emociones, las creencias, los intereses y los valores.

Por su parte, Marzano, Pickering y Debra (2005) señalan que la motivación puede ser impulsada a través de acciones concretas que el docente realice con estrategias que les permitan a los estudiantes sentir bienestar al momento de estar aprendiendo.

\section{Segunda dimensión: La comprensión}

Esta dimensión es entendida como la actividad en la que se establecen relaciones entre el nuevo contenido y los elementos ya disponibles en su estructura cognitiva (Coll, 2007; Marín, 2015); es decir, que los estudiantes vayan comprendiendo el material o la información que se trabaja en clases (Zarzar, 2000). Aquí se construyen significados y se trata de una concordancia muy especial, pues es sustantiva (de significado), es a lo que frecuentemente se le llama comprensión de los contenidos, porque en él solamente se llega a conocer el objeto de estudio.

Es decir, este es el momento en que los estudiantes se aventuran a exponer suposiciones y las contrastan con el doble objetivo de remover el conocimiento actual e incitarse a utilizar la fuerza para intentar dar respuesta a las cuestiones que se han planteado (Zabala, 2005); esto se ve reflejado en la necesidad de asegurar la construcción de estos aprendizajes significativos, relacionando el nuevo material de forma significativa y no arbitraria e integrarlo en la estructura cognitiva previa del estudiante. Si no se alcanza esta conexión, sería un aprendizaje meramente memorístico (Tejada, 2008).

\section{Tercera dimensión: La funcionalidad}

La funcionalidad por su parte refiere que los conocimientos adquiridos puedan ser efectivamente utilizados (Coll, 2014). En este sentido se puede señalar que cuanto más numerosas y complejas sean las relaciones establecidas entre el nuevo contenido de aprendizaje y los elementos de la estructura cognitiva y cuanto más profunda sea su asimilación, mayor será su grado de significatividad y por lo tanto mayor la funcionalidad que el estudiante podrá darle a los conocimientos en nuevos contenidos y situaciones. Por su parte, Riveros (2009) coincide en que el aprendizaje es funcional en el sentido de que los nuevos contenidos asimilados están disponibles para ser utilizados para resolver problemas en contextos diferentes; de tal manera que el aprendizaje más efectivo se da cuando los alumnos desarrollan un entendimiento a profundidad del conocimiento.

De tal forma que una vez que el estudiante está motivado, comprende el material y le encuentra funcionalidad, estará en condiciones de presentarse la siguiente dimensión del aprendizaje significativo:

\section{Cuarta dimensión: La participación activa}

Esta dimensión es reconocida como el estudio, análisis, discusión y elaboración de la información recibida (Zarzar, 2000). Implica el compromiso para llevar a cabo un estudio sistemático, analítico y reflexivo del contenido (Marín, 2015).

Dicha participación se empieza a dar cuando el estudiante asume un papel activo y trabaja sobre la información recibida, es decir, el estudiante deberá integrar diferentes momentos en los que reflexione sobre su propio proceso cognitivo (Garcés Suárez y Alcívar, 2016), 
que analice las razones que le han llevado a realizar las actividades de aprendizaje, que revise sus experiencias anteriores, que valore la eficacia de los instrumentos utilizados, su actuación y detecte las dificultades que ha hallado y los medios para resolverlos, además de que extraiga conclusiones que le sirvan para afrontar otros retos de aprendizaje (Álvarez-Flores y Núñez Gómez, 2014).

\section{Quinta dimensión: La relación con la vida real}

Cuando se habla de la relación con la vida real, se refiere a la aplicación de la información recibida para la solución de problemas reales o posibles (Zarzar, 2000). Es decir, la satisfacción de necesidades reales para los estudiantes a través del material y/o información que ha recibido es una de las dimensiones más alta y que reflejan un grado de significatividad mayor; en este sentido se presentan situaciones donde el estudiante debe poner a prueba sus conocimientos anteriores, construyendo relaciones sustantivas para usarlas en nuevos contextos, en los cuales se exige creatividad para la resolución de situaciones problemáticas. Para tal efecto será necesario que el estudiante disponga de una estructura cognoscitiva, es decir, haber pasado por las dimensiones anteriores y que le sirvan para dar respuesta al número más elevado posible de problemas y cuestiones que la vida en sociedad les plantee.

\subsection{Las tecnologías y el proceso de enseñanza aprendizaje}

Ahora bien, estas dimensiones pueden ser impulsadas a través de herramientas que apoyen el proceso de enseñanza aprendizaje, como es el caso de las tecnologías; por lo que aquí radica la importancia de la mediación tecnológica, la cual tiene su raíz con Lev Vygotsky (2002), quien se considera un referente imprescindible, ya que aporta claves para hacer propuestas en el análisis de las funciones que asumen los medios en los procesos de enseñanza y aprendizaje.

Por lo tanto, las TIC al mediar la relación educativa la transforman; esta modificación se genera a partir de una estructura básica de acción tecnológica que facilita el procesamiento, gestión y distribución de la información, agregando a la relación educativa, nuevas posibilidades y limitaciones para el aprendizaje (Suárez, 2011); posicionando a las TIC como herramientas e instrumentos que sirven como mediadores entre la enseñanza y el aprendizaje, entendiendo que su función es la de facilitar la actividad de aprendizaje.

Sustentados en sus investigaciones, Guglietta (2011) y Fainholc (2004, 2010) y Fainholc, Zangara y Grassis (2013) han señalado que el aprendizaje se facilita con la mediación o interacción con los otros: el diálogo, el debate y la negociación son estrategias potenciadoras del aprendizaje. De igual forma, tal como lo manifiesta Murillo, MartínezGarrido y Hernández-Castilla (2011) una parte importante para que los estudiantes aprendan mejor es la motivación que se les pueda brindar por parte del docente, esto a través de nuevos estímulos o formas de interactuar; es decir, cuando el profesor utiliza diferentes actividades los contenidos se perciben como más interesantes y estimulantes provocando su natural curiosidad.

Por tal motivo la tecnología se convierte no sólo en un vehículo para la evolución, sino en un catalizador de transformaciones; enfatizando que la mediación tecnológico-educativa deja de ser instrumental para convertirse en parte de la estructura de esta nueva civilización llamada Sociedad del Conocimiento, desde un lugar dentro de la cultura, llevado adelante por los programas educativos formales, no formales e informales que hoy utilizan materiales educativos de todo tipo, aunque preferentemente con las TIC. 
De tal manera que para que en la parte académica el estudiante pueda ser apoyado en el desarrollo de habilidades y competencias es necesario que el docente afirme el aprendizaje con acciones específicas, es decir, con estrategias de enseñanza, y que para este caso en particular se son las conocidas como mediadas por tecnología las cuales se aplican en los entornos virtuales de formación, entendidos como herramientas que permiten superar limitaciones de interactividad inherentes a las tradicionales prácticas en la educación a distancia (Begoña, 2009).

Por tanto, la implementación de dichas tecnologías en la educación ha dado paso a distintas modalidades de formación entre las cuales destaca la mixta (blended o b-learning), que es aquella que tiende a generar una interacción directa entre los sistemas presenciales y la educación a distancia. Esta modalidad se ha fortalecido debido a la enorme demanda de nuevas técnicas y el incremento en el uso de Internet, así como a la utilidad que esto ha dado en todos los ámbitos de estudio.

Aunque algunos autores, como Bartolomé (2004) aseguran que para hablar del surgimiento del blended learning habría que discutir del fracaso del e-learning, es difícil sostener tal afirmación de manera plena, habida cuenta que hay otros itinerarios que explican el devenir de esta modalidad; pues, aunque los estudios realizados por estos autores han demostrado el éxito en modalidades mixtas, también es cierto que en ninguno de ellos ha señalado convenientemente el fracaso de la educación virtual. Asimismo, existen otras investigaciones, como por ejemplo la de Osbaldo (2010) y González (2011), que concluyen que la modalidad blended learning es plenamente aceptada y que las universidades la han adoptan como parte de sus modalidades de estudio. En tal sentido, resulta importante señalar que la modalidad mixta o también llamada blended learning, no es estática, su naturaleza y papel se hayan inexorablemente unidos a las expectativas que tenemos para los estudiantes y los profesores.

En este estudio se pretende conocer la percepción que los estudiantes universitarios tienen respecto al nivel de aprendizaje significativo que alcanzan al utilizar estrategias de enseñanza impuestas por sus docentes en una modalidad mixta; para el cumplimiento de tal objetivo se diseñó y validó un instrumento de medida denominado Escala de Percepción de Aprendizaje Significativo en Blended Learning (E.P.A.S.B.L.) y que pretendía medir las cinco dimensiones del aprendizaje significativo en relación con las actividades presentadas por el profesor dentro de la plataforma Moodle para el desarrollo de las clases.

\section{Método}

Diseño

El enfoque utilizado en la investigación fue de corte cuantitativo con un diseño no experimental y transversal y de alcance descriptivo-correlacional. Además, utilizando la misma metodología cuantitativa, en este trabajo se determinaron las propiedades psicométricas de un instrumento para medir la percepción de los estudiantes respecto del aprendizaje significativo obtenido en la enseñanza blended learning.

\section{Muestra}

La muestra fue de tipo no probabilística incidental. Los participantes en el estudio fueron 110 alumnos de la carrera profesional de abogado que estudian los semestres de segundo, 
cuarto, sexto y octavo en una modalidad mixta de una universidad pública mexicana. El $79,2 \%$ de los participantes fueron del sexo masculino y el $20,8 \%$ del femenino.

\section{Instrumento}

Para llevar a cabo la investigación se confeccionó una escala cuyo nombre fue Escala de Percepción de Aprendizaje Significativo en Blended Learning (E.P.A.S.B.L.), la cual en su versión definitiva quedó integrada por un total de 10 ítems, mismos que a su vez se agruparon en 3 factores correlacionados. El formato de dicho instrumento es de tipo Likert con cinco opciones de respuesta, las cuales son: nunca; la mayoría de las veces no; algunas veces sí-algunas veces no; la mayoría de las veces sí; y siempre.

\section{Procedimiento}

El diseño y validación de la escala transitó por diferentes momentos, el primero consistió en una revisión teórica-científica sobre el constructo teórico en cuestión; en dicho proceso se reconoció que no existía un instrumento que midiera la percepción del aprendizaje significativo que tienen los estudiantes en una modalidad mixta. En razón de ello, se pasó a un segundo momento el cual consistió en elaborar los correspondientes reactivos. En dicho momento se construyeron un total de 71 ítems que de manera inicial pretendían medir cinco dimensiones del aprendizaje significativo, y en las cuales se sustentó teóricamente este estudio: motivación ( 8 ítems), comprensión ( 17 ítems), funcionalidad (19 ítems), participación activa (18 ítems) y relación con la vida real (9 ítems).

Posteriormente se pasó a un tercer momento, en el cual se sometió el instrumento a la validación de contenido. Para tal efecto se consultó a 7 profesores universitarios que fungieron en calidad de expertos. Como resultado de ello se efectuaron adecuaciones a los ítems. En un cuarto momento y con la intención de evaluar la comprensión de los ítems, se llevó a cabo la denominada prueba piloto con veinte estudiantes. Como consecuencia del tal proceso se llevaron a cabo algunas correcciones a la redacción de los reactivos considerados.

En el quinto momento, se aplicó la escala a los 110 estudiantes antes referidos. Enseguida y con la intención de revelar el poder discriminativo de los ítems, en el momento seis, se instrumentaron una serie de pruebas t para comparar las medias obtenidas por cada uno de los ítems de los sujetos de los grupos "bajo" (27\% de sujetos con las puntuaciones más bajas) y "alto" (27\% de sujetos con las puntuaciones más altas). Producto de ello se anotó que el total de los ítems fueron altamente discriminativos.

Posteriormente, en el momento siete, se pasó al proceso de validación de constructo, para tal efecto se ejecutó tanto el análisis factorial exploratorio (con la primera mitad de la muestra) como el confirmatorio (segunda mitad). Los resultados de dichos análisis aparecen en el apartado de resultados.

Para finalizar, se calculó la confiabilidad de la escala, se obtuvieron las medias y se identificaron las frecuencias. Para reconocer diferencias en la percepción de aprendizaje significativo por semestre que cursan los estudiantes, se utilizó el análisis de varianza (ANOVA) unidireccional o de un factor (one-way); para el caso de las diferencias de la variable de interés conforme el género y rangos de edad se emplearon pruebas t de student para muestras independientes. Tales datos se obtuvieron mediante el uso del paquete estadístico SPSS, 22.0 


\section{Resultados}

$\mathrm{El}$ análisis factorial exploratorio se efectuó permitiendo la estructura libre de factores, empleando el procedimiento de componentes principales con rotación varimax. El $\mathrm{KMO}=0,782$ y la prueba de esfericidad de Barlett $(\mathrm{p} \leq \mathrm{OOO})$ evidenciaron un modelo apropiado y sin esfericidad.

Para que los ítems o reactivos formaran parte de los factores se tomaron en cuenta los criterios siguientes: a) selección de aquellos ítems con una saturación igual o superior a 0,60 en la matriz factorial rotada y b) elección de factores que contenían al menos tres ítems, c) se eliminó el ítem 47 "Tiene actividades que presentan diversidad de puntos de vista y de perspectivas sobre los temas tratados en el curso, a fin de reflexionar y debatir sobre los mismos", porque reportó con carga compartida (factores 2 y 3) y d) se excluyeron ítems que aun teniendo la saturación requerida no tenían congruencia teórica con los demás reactivos que integraban al factor.

Con tales referentes se aislaron un total de 3 factores de primer orden con valor propio igual o superior a 1, que explicaron el $60 \%$ de la varianza total. Con base al contenido de los ítems que conformaban los factores se les nombró como "Comprensión", "Participación Activa" y "Funcionalidad y Relación con la Vida Real". En cuadro 1 aparecen los ítems, las saturaciones, comunalidades, varianzas y valor propio de cada uno de los factores.

El siguiente paso consistió en confirmar la estructura factorial mediante un análisis factorial confirmatorio usando el Structural Equation Modeling Software (EQS, versión 6.2). El modelo evaluado constó de 3 factores independientes (cuadro 1) con 7 reactivos en el primer factor, 5 con el segundo y 3 en el tercero. Se trazaron además otros dos modelos, uno con un solo factor que integraba a todos los ítems señalados en el cuadro 1 y uno más con tres factores correlacionados. Cabe señalar que en el último modelo se decidió eliminar los ítems 10,38, 41, 51 y 49, debido a su ambigüedad factorial, ya que saturan significativamente en más de un factor.

La bondad de ajuste de los modelos señalados se evaluó mediante los indicadores siguientes. Ji cuadrada $X^{2}$, el promedio de los residuales (RMR), los residuales estandarizados (RMSEA), el índice de bondad de ajuste (GFI) y el índice de ajuste comparativo (CFI).

Los últimos cuatro índices son de los más usados ya que están menos afectados por el tamaño de la muestra (García, Gallo y Miranda, 1998). Para que existan ajustes aceptables, los valores CFI y GFI deberían superar el valor de 0,90 (cuanto mayor sea el valor, mejor ajuste). Con relación al RMR los valores cercanos a cero indican buen ajuste y menores de 0,10 se consideran aceptables. Los valores RMSEA deben ser menores a 0,08 para tener un ajuste aceptable de acuerdo con Browne y Cudeck (1993), o cercanos a 0,05 para obtener un buen ajuste según Byrne (2001). Respecto a la interpretación del cociente $X^{2}$, se considera que no debe ser significativa. Los índices de bondad de ajuste de cada modelo están referidos en el cuadro 2. 
Cuadro 1. Cargas factoriales, comunalidades y varianza

\begin{tabular}{|c|c|c|c|}
\hline $\mathbf{N}^{\circ}$ & İTEM & SAT. & CoMun. \\
\hline \multicolumn{4}{|c|}{ Factor 1. Comprensión } \\
\hline 23 & $\begin{array}{l}\text { Plantea preguntas, actividades, vínculos u otros medios diversos } \\
\text { para establecer relaciones entre mis conocimientos. }\end{array}$ & 0,720 & 0,756 \\
\hline 33 & $\begin{array}{l}\text { Es breve y concreto ampliando y profundizando los contenidos en } \\
\text { páginas complementarias }\end{array}$ & 0,711 & 0,617 \\
\hline 32 & $\begin{array}{l}\text { Cuenta con contenidos que aprendo durante el curso y que son } \\
\text { tomados en cuenta como material de trabajo en las actividades. }\end{array}$ & 0,693 & 0,826 \\
\hline 35 & $\begin{array}{l}\text { Tiene información adecuada a mis conocimientos y mis necesidades } \\
\text { de las tareas a realizar en cada actividad }\end{array}$ & 0,689 & 0,538 \\
\hline 12 & $\begin{array}{l}\text { Incluye recursos específicos para relacionar los nuevos contenidos } \\
\text { de la materia con mis conocimientos iniciales. }\end{array}$ & 0,674 & 0,707 \\
\hline 10 & $\begin{array}{l}\text { Tiene en cuenta mis conocimientos iniciales a la hora de plantear y } \\
\text { dinamizar las actividades para promover los aprendizajes }\end{array}$ & 0,626 & 0,752 \\
\hline \multirow[t]{2}{*}{38} & $\begin{array}{l}\text { Tiene contenidos con conceptos, procedimientos y actitudes en } \\
\text { correspondencia con los objetivos prioritarios del curso y con las } \\
\text { demandas de las tareas a realizar. }\end{array}$ & 0,611 & 0,653 \\
\hline & $\%$ de varianza explicada 43,95 & & \\
\hline \multicolumn{4}{|c|}{ Factor 2. Participación Activa } \\
\hline 41 & $\begin{array}{l}\text { Tiene actividades que promueven la intervención con mis } \\
\text { compañeros en la selección de nuevos contenidos y/o actividades } \\
\text { de acuerdo con mis intereses }\end{array}$ & 0,793 & 0,832 \\
\hline 46 & $\begin{array}{l}\text { Hay actividades que me permiten reflexionar sobre lo que he } \\
\text { aprendido y ahora se. }\end{array}$ & 0,694 & 0,754 \\
\hline 51 & $\begin{array}{l}\text { Cuenta con actividades que me permiten desempeñar diversos roles } \\
\text { activos dentro de una dinámica de grupo. }\end{array}$ & 0,673 & 0,840 \\
\hline 29 & $\begin{array}{l}\text { Introduce preguntas en este sentido: ¿Qué piensas sobre lo } \\
\text { expresado? ¿Qué experiencias personales tienes al respecto? }\end{array}$ & 0,671 & 0,712 \\
\hline \multirow[t]{2}{*}{13} & $\begin{array}{l}\text { Promueve la reflexión sobre mis experiencias personales anteriores } \\
\text { al plantear una nueva experiencia. }\end{array}$ & 0,653 & 0,750 \\
\hline & $\%$ de varianza explicada 9,45 & & \\
\hline \multicolumn{4}{|c|}{ Factor 3. Funcionalidad y Relación con la Vida Real } \\
\hline 48 & $\begin{array}{l}\text { Tiene actividades que me invitan a utilizar y contrastar fuentes de } \\
\text { información, argumentos de fundamentación, entre otros. }\end{array}$ & 0,799 & 0,739 \\
\hline 49 & $\begin{array}{l}\text { Tiene actividades dirigidas a promover la elaboración de planes de } \\
\text { actuación personal o colectiva y la toma de decisiones al respecto. }\end{array}$ & 0,792 & 0,797 \\
\hline \multirow[t]{2}{*}{37} & $\begin{array}{l}\text { Promueve el contraste de informaciones procedentes de distintas } \\
\text { fuentes de información, para poner a prueba su validez y } \\
\text { relevancia. }\end{array}$ & 0,700 & 0,798 \\
\hline & $\%$ de varianza explicada 6,59 & & \\
\hline
\end{tabular}

Nota: Matriz de cargas factoriales, comunalidades y varianza explicada de cada uno de los factores. Fuente: Elaboración propia.

Como se aprecia el modelo mejor ajustado es tres (tres factores correlacionados con 10 ítems), aunque el GFI, no alcanza un valor mayor a 0,90 el resultado encontrado se ubica en estándares aceptables. 
Cuadro 2. Índices de bondad de ajuste de cada uno de los modelos propuestos

\begin{tabular}{lccccc}
\hline \multicolumn{1}{c}{ MODELO FACTORIAL } & $\mathbf{X}^{2}$ Y P & CFI & GFI & RMR & RMSEA \\
\hline Un factor & 136,91 y 0,00 & 0,92 & 0,74 & 0,09 & 0,09 \\
Tres factores independientes & 302,17 y 0,00 & 0,64 & 0,63 & 0,69 & 0,20 \\
Tres factores correlacionados 10 ítems & 43,397 y 0,09 & 0,97 & 0,86 & 0,06 & 0,08 \\
\hline
\end{tabular}

Fuente: Elaboración propia.

La confiablidad del instrumento, medida por alfa de Cronbach, fue de $\alpha=0,871$ para el factor de Comprensión, de $\alpha=0,826$, para el de Participación Activa y $\alpha=0,795$ para el factor Funcionalidad y Relación con la Vida Real y el total fue $\alpha=0,917$. Con tales resultados se puede aseverar que la confiablidad de la escala es aceptable.

En síntesis, los aspectos antes referidos indican que la E.P.A.S.B.L. mostró propiedades psicométricas adecuadas, siendo válida y confiable para ser empleada en la actual investigación.

\subsection{Percepción del aprendizaje significativo de estudiantes en blended learning}

En seguida se muestran los resultados obtenidos con la aplicación de la E.P.A.S.B.L. en estudiantes de la carrera de Abogado en una modalidad mixta. Los resultados presentados en el cuadro 3 indican que en general la percepción de aprendizaje significativo de los participantes puede interpretarse como moderado $(\overline{\mathrm{x}}=3,68)$, que no corresponde a baja ni alta percepción de aprendizaje significativo.

Cuadro 3. Percepción del aprendizaje significativo

\begin{tabular}{ccccc}
\hline NÚMERo De PARTICIPANTES & PUNTUACIÓN Mínima & PUNTUACIÓN MÁXIMA & $\overline{\mathbf{X}}$ & DE \\
\hline 110 & 1 & 5 & 3,68 & 0,854 \\
\hline
\end{tabular}

Fuente: Elaboración propia.

Enseguida para poder determinar el uso de pruebas paramétricas, se consideraron los criterios de normalidad y el de homocedasticidad. A través del estadístico de Kolmogorov se identificó la distribución normal de la variable percepción de aprendizaje significativo y por medio de Levene se identificó la homocedasticidad entre las varianzas de los distintos grupos a comparar.

Para detectar diferencias en la percepción de aprendizaje significativo conforme al género de los estudiantes, se aplicó la prueba estadística t de Student. Los resultados de $t(107)=$ $-1,878$ y $p=0,716$, muestran que no existen diferencias entre lo que perciben los hombres y las mujeres.

Cuadro 4. Medias de la percepción de aprendizaje significativo de los estudiantes por género

\begin{tabular}{cccc}
\hline GÉNERO & No. ESTUDIANTES & $\overline{\mathbf{X}}$ & DE \\
\hline Femenino & 16 & 3,28 & 1,020 \\
Masculino & 61 & 3,75 & 0,847 \\
\hline
\end{tabular}

Fuente: Elaboración propia.

Con relación a las diferencias en la percepción de aprendizaje significativo por semestre de los estudiantes, con la ayuda de ANOVA se evidenció que estadísticamente tampoco es significativa la diferencia, $\mathrm{F}(3,106)=0,515 \mathrm{y} \mathrm{p}=0,673$ 
Cuadro 5. Medias de la percepción de aprendizaje significativo por semestre

\begin{tabular}{cccc}
\hline SEMESTRE & No. ESTUDIANTES & $\overline{\mathbf{X}}$ & DE \\
\hline 2 & 19 & 3,84 & 0,895 \\
4 & 19 & 3,49 & 0,832 \\
6 & 41 & 3,66 & 0,782 \\
8 & 31 & 3,71 & 1,012 \\
Total & 110 & 3,68 & 0,874 \\
\hline
\end{tabular}

Fuente: Elaboración propia.

Por último, para identificar diferencias en la percepción de aprendizaje significativo por rangos de edad de los estudiantes, se utilizó nuevamente la t de Student. Mediante tal prueba de hipótesis se reconoció la existencia diferencias significativas, $\mathrm{t}(107)=-2,151$ y $\mathrm{p}=0,034$.

Cuadro 6. Medias de la percepción de aprendizaje significativo por rangos de edad

\begin{tabular}{cccc}
\hline RANGO DE EDAD* & No. EsTUDIANTES & $\overline{\mathbf{X}}$ & DE \\
\hline 1 (18 a 25 años) & 42 & 3,45 & 0,894 \\
2 (25 a 60 años) & 68 & 3,82 & 0,838 \\
\hline
\end{tabular}

Nota: Los rangos de edad se definieron conforme las etapas evolutivas del desarrollo psicosocial de Erick Erikson (Bordignon, 2005), a saber, adultez temprana entre los 18 y 25 años y adultez media entre los 25 y 60.

Fuente: Elaboración propia.

\section{Discusión y conclusiones}

En primer lugar, conviene destacar que la E.P.A.S.B.L. puede utilizarse con confianza, ya que de los factores encontrados representan los constructos que se pretenden medir y que su aplicación repetida en los mismos sujetos presenta resultados semejantes. Sin embargo, para que sus propiedades puedan generalizare es preciso que se aplique en otras poblaciones y contextos.

Con relación a los hallazgos, se puede señalar que los resultados revelan que existe una percepción media de aprendizaje significativo en la población estudiada, lo que podría interpretarse como que los estudiantes no perciben grandes aprendizajes en dicha modalidad, pero tampoco sostienen la idea contraria. Tal vez ello podría explicarse por lo señalado por Díaz Barriga (2010) en el sentido de que todavía los profesores adolecen de competencias suficientes para generar oportunidades de aprendizaje que ayuden a los alumnos a cuestionarse respecto de los que ya conocen, buscar nueva información, y trabajar en colaboración con otros para resolver problemas y tomar decisiones.

Por otra parte, la condición de que no se advirtieran diferencias en la percepción de aprendizaje significativo entre los distintos semestres y por género, sugiere que tales condiciones no son variables que puedan explicar las diferencia en el aprendizaje, ya que este depende más de los aspectos que autores como Ausubel (2002) han sostenido, a saber, que el material empleado por los profesores sea potencialmente significativo (con estructura lógica que facilite la comprensión), que el estudiante tenga disposición para el aprendizaje (motivación) y que el conocimiento previo de cada estudiante posee pueda ser relacionado con el conocimiento nuevo. Este resultado, pues, corroboró que los medios, representados en este caso por las tecnologías digitales, por sí solos, no determinan los 
logros en el aprendizaje tal como lo encontrado en las investigaciones realizadas por Francisco (2006) y Hernández, Pérez y García (2001).

Con lo anterior se muestra que la efectividad de las tecnologías depende de la pertinencia de las estrategias utilizadas con respecto al tipo de aprendizaje a desarrollar; es decir, la selección de estrategias mediadas con tecnología debe considerar de qué manera se quiere influir en el aprendizaje y a partir de ellas determinar qué recursos son los más adecuados para alcanzarlos. Es decir, el docente debe realizar una planeación didáctica que tenga impacto en el aprendizaje significativo, pues esto constituirá el patrón que permita enfrentar de forma ordenada y congruente las situaciones a las que el estudiante se enfrentará en su vida profesional o cotidiana y en el caso del profesor, a su práctica docente (Ascencio, 2016) De tal manera que tal como lo señalan Garrote Rojas, Garrote Rojas y Jiménez Fernández (2016) "Los docentes debemos trasmitir a los estudiantes los contenidos de las diferentes asignaturas a través de una metodología que les resulte atractiva, así les facilitaremos la adquisición de los mismos" (p. 37).

Por otro lado y con relación a las diferencias encontradas en la percepción de aprendizaje significativo conforme a los distintos rangos de edad de los estudiantes, se puede inferir que tal condición probablemente ocurra en razón de que las personas mayores de 25 años frecuentemente cuentan ya con un empleo y otras responsabilidades personales, por lo que la decisión de estudiar una carrera profesional implica para ellos una mayor motivación personal, pero sobre todo, la búsqueda de la aplicabilidad y funcionalidad en sus trabajos de los conocimientos adquiridos. Además, debido a su mayor edad, es factible pensar que cuenten también con mayores conocimientos previos.

Por su parte y en materia de las limitaciones de la presente investigación, es importante destacar su naturaleza descriptiva y correlacional, no permite establecer inferencias causales sobre la percepción de aprendizaje significativo en la modalidad blended learning; además de que los resultados obtenidos emergen de auto-informes, de tal manera que se pueden presentar respuestas sesgadas ya que es el propio participante quien informa de sus conductas y actitudes. Por ello y para una mayor profundización, serán necesarias investigaciones de corte o alcance explicativo y/o de estudios con enfoque cualitativo o comprensivo. Asimismo, se requiere que en futuros trabajos se disponga de diferentes informantes que den cuenta del proceso de aprendizaje de los alumnos, como por ejemplo de profesores y tutores y que dicha información pueda ser contrastada con el presente estudio.

Ante estas condiciones se considera conveniente seguir indagando sobre el estado que guardan las modalidades educativas que son mediadas por las nuevas tecnologías, de manera particular sobre la relación que guardan las mismas con respecto al aprendizaje de los estudiantes, pues esto representan una de las prioridades y metas para el desarrollo de la educación vía las TIC, sin embargo, no existen suficientes investigaciones que corroboren estas acciones. De lo anterior también se desprende que existen retos significativos para la adopción de la tecnología ya que la mayoría de los académicos no están utilizando las TIC de manera significativa para el aprendizaje y la enseñanza, ni para organizar sus investigaciones. Es decir, los profesores no utilizan la tecnología con la visión de generar aprendizajes significativos en el estudiantado, situación que debe considerarse como un foco de atención para las instituciones educativas.

Finalmente, a manera de cierre, conviene expresar la importancia de ampliar las investigaciones en otros contextos; sería prudente realizar un mayor número de estudios 
en torno a cómo las TIC han impactado verdaderamente en la calidad de los aprendizajes que se generan en las distintas modalidades educativas de nuestro medio y de otros contextos.

\section{Referencias}

Ausubel, D. (2002). Adquisición y retención del conocimiento. Una perspectiva cognitiva. Barcelona: Paidós.

Aguaded, I. y Cabero Almenara, J. (2014). Avances y retos en la promoción de la innovación didáctica con las tecnologías emergentes e interactivas. EDUCAR, 50, 67-83. https://doi.org/10.5565/rev/educar.691

Álvarez-Flores, E. y Núñez Gómez, P. (2014). Uso de las redes sociales como elemento de interacción y construcción de contenidos en el aula: Cultura participativa a través de facebook. Historia $y \quad$ Comunicación Social, $18, \quad$ 53-62. https://doi.org/10.5209/rev_HICS.2013.v18.44225

Ascencio, C. (2016). Adecuación de la planeación didáctica como herramienta docente en un modelo universitario orientado al aprendizaje. REICE. Revista Iberoamericana sobre Calidad, Eficacia y Cambio en Educación, 14(3), 109-130. https://doi.org/10.15366/reice2016.14.3.006

Ball, C. T. y Pelco, L. E. (2006). Teaching research methods to undergraduate psychology students using an active cooperative learning approach. International Journal of Teaching and Learning in Higher Education, $17(2), 147-154$.

Bartolomé, A. (2004). Blended learning. Conceptos básicos. Pixel-Bit. Revista de Medios y Educación, 23, 7-20.

Begoña, M. (2009). Educación y nuevas tecnologías. Educación a distancia y educación virtual. Revista de Teoría y Didáctica de las Ciencia, 9, 209-222.

Bos, M., Ganimian, A. y Vegas, E. (2015). América Latina en Pisa 2012: ¿Cómo se relaciona el aprendizaje estudiantil con los recursos que se invierten en educación? Recuperado de https://publications.iadb.org/handle/11319/6440

Browne, M. W. y Cudeck, R (1993). Alternative ways of assessing model fit. En K. Bollen y J. Long (Eds.), Testing structural equation models (pp. 136-162). Newbury Park, CA: Sage.

Byrne, B. M. (2001). Structural equation modeling with AMOS: Basic concepts, applications, and programming. Mahwah, NJ: Lawrence Erlbaum.

Coll, C. (2007). El constructivismo en el aula. Madrid: Graó.

Coll, C. (2014). El sentido del aprendizaje hoy: Un reto para la innovación educativa. Aula de Innovación Educativa, 232, 12-17.

Díaz Barriga, A. (2005). El profesor de educación superior frente a las demandas de los nuevos debates educativos. Perfiles Educativos, 27(108), 9-30.

Díaz Barriga, F. B. (2010). Estrategias docentes para un aprendizaje significativo. Ciudad de México: Mc Graw Hill.

Espinoza, J. (2017). Los recursos didácticos y el aprendizaje significativo. Espirales. Revista Multidisciplinaria de Investigación, 2, 33-38.

Fainholc, B. (2004). La calidad en la educación a distancia continúa siendo un tema muy complejo. Revista de Educación a Distancia, 12, 1-7. 
Fainholc, B. (2010). La formación científico-tecnológica digital en educación superior. Revista de Universidad y Sociedad del Conocimiento, 7(2), 1-23.

Fainholc, B., Zangara, A. y Grassis, C. (2013). Modelo de enseñanza mediado para el desarrollo profesional docente de estudiantes en formación, con énfasis en el trabajo colaborativo virtual. Recuperado de http://hdl.handle.net/10915/26816

Francisco, J. (2006). Efectos de una estrategia instruccional mediada por tecnologías digitales sobre el desempeño estudiantil. Compendiu, 9(17), 5-19.

García, E., Gallo, P. y Miranda, R. (1998) Bondad de ajuste en el análisis factorial confirmatorio. Psicothema, 10, 717-724.

Garcés Suárez, E. y Alcívar, O. (2016). Las tecnologías de la información en el cambio de la educación superior en el siglo XXI: Reflexiones para la práctica. Revista Universidad y Sociedad, 8(4), 171-177.

García-Valcárcel, A. y Tejedor Tejedor, F. J. (2017). Percepción de los estudiantes y el valor de las TIC en sus estrategias de aprendizaje y su relación con el con el rendimiento. Educación XXI, 2O(2), 137-159. https://doi.org/10.5944/educxx 1.19035

Garrote Rojas, D., Garrote Rojas, C. y Jiménez Fernández, S. (2016). Factores influyentes en motivación y estrategias de aprendizaje en los alumnos de grado. REICE. Revista Iberoamericana sobre Calidad, Eficacia y Cambio en Educación, 14(2), 31-44. https://doi.org/10.15366/reice2016.14.2.002

Garnham, C. y Kaleta, R. (2002). Introducction to hybrid courses. Teaching with technology today. Recuperado de http://www.uwsa.edu/ttt/articles/garnham.htm

González, M. (2011). Aprendizaje interactivo en la enseñanza de estadística a través de sitios web con metodología B-learning. Un estudio empírico. Alicante: Universidad de Alicante.

González, K., Padilla, J. y Rincón, D. (2012). Formación del docente en contextos b-learning: Implicaciones tecnológicas, investigas y humanísticas. Revista Virtual Universidad Católica del Norte, 36(1), 48-74.

Guglietta, L. (2011). Instituto internacional para la educación superior de América Latina y el Caribe. Recuperado de http://www.iesalc.unesco.org.ve/index.php

Hernández, M. Pérez, A. y García, B. (2001). Influencia de las estrategias instruccionales basadas en simulaciones sobre el nivel de aprendizaje. Revista Científica Teorías, Enfoques y Aplicaciones en las Ciencias Sociales, 3(6), 71-84.

Hidalgo, N. y Murillo, F. (2017). Las concepciones sobre el proceso de evaluación del aprendizaje de los estudiantes. REICE. Revista Iberoamericana sobre Calidad, Eficacia y Cambio en Educación, 15(1), 107-128. https://doi.org/10.15366/reice2017.15.1.007

Islas-Torres, C. (2015). La práctica del estudiante en el B-learning: Una observación sistémica. Revista Dínnovació $i$ Recerca en Educació, 38, 43-61.

Laster, S. (2005). Redefining blended learning. En D. Laurillard (Ed.), Rethinking university teaching (pp. 134-156). Londres: Routledge.

Marcelo, C., Yot, C., Murillo, P. y Mayor, C. (2016). Actividades de aprendizaje con tecnologías en la universidad. ¿Qué uso hacen los profesores? Profesorado. Revista de Currículum y Formación de Profesorado, 20(3), 283-312.

Marín, E. (2015). Aprendizaje constructivista para el análisis de estructuras mediante el uso de un entorno virtual. Revista Tecnocientífica URU, 9, 41-49.

Marzano, R., Pickering, J. y Debra, J. (2005). Dimensiones del aprendizaje. Tlaquepaque: ITESO. 
Monereo, C. (2013). Aprendizaje estratégico y tecnologías de la información y la comunicación: Una revisión crítica. Teoría de la Educación, 4(1) 15-41.

Murillo, F. J., Martínez-Garrido, C. y Hernández-Castilla, R. (2011). Decálogo para una enseñanza eficaz. REICE. Revista Iberoamericana sobre Calidad, Eficacia y Cambio en Educación, 9(1), 627.

OEI. (2015). Metas educativas 2021. Madrid: Organización de Estados Iberoamericanos.

Osbaldo, T. (2010). Contexto y desarrollo de la modalidad educativa blenden learning en el sistema universitario iberoamericano. Revista Mexicana de Investigación Educativa, 15(45), 345-370.

Peña, J. A. (2010). Concepciones de enseñanza cara a cara, mixta y en línea de profesores universitarios. Apertura, 2(1), 36-47.

Pérez Gómez, A. (2008). ¿Competencias o pensamiento práctico? La construcción de los significados de representación y de acción. Madrid: Morata.

Pérez Sánchez, L. y Beltrán Llera, J. (2014). Estrategias de aprendizaje: Función y diagnóstico en el aprendizaje del adolescente. Padres y Maestros, 358, 34-38. https://doi.org/10.14422/pym.voi358.4086

Riveros, X. (2009). Constructivismo: elementos conceptuales. Santiago de Chile: Universidad de Chile.

Suárez, C. (2011). Los entornos virtuales de aprendizaje como instrumentos de mediación. Recuperado de http://campus.usal.es/ teoriaeducacion/rev_numero_04/n4_art_suarez.htm

Tejada, L. (2008). Aprendizaje significativo en el aula. Papeles de Educación, 44(1),127-144.

UNESCO. (2014). Documento de posición sobre la educación después de 2015. Recuperado de http://unesdoc.unesco.org/images/o022/002273/227336s.pdf

Vigotsky, L. (2000). El desarrollo de los procesos psicológicos superiores. Barcelona: Crítica.

Zabala, A. (2005). Enfoque globalizador y pensamiento complejo, una respuesta para la comprensión e intervención en la realidad. Madrid: Graó.

Zarzar, C. (2000). La didáctica grupal. Ciudad de México: Progreso.

\section{Breve CV de los autores}

\section{María del Rocío Carranza Alcántar}

Profesora investigadora de la Universidad de Guadalajara, México, con Perfil Deseable por parte del Programa para el Desarrollo del Profesorado (PRODEP), Maestra en Enseñanza de las Ciencias y Doctora en Sistemas y Ambientes Educativos; forma parte del grupo de investigación en consolidación "Educación y Sociedad", actualmente cuenta con el reconocimiento como investigador Nivel 1 que otorga el Sistema Nacional de Investigadores del CONACYT. La principal línea de investigación que trabaja está relacionada con el uso de las tecnologías para la educación. ORCID ID: 0000-0003-14109130. Email: mcarranza@cualtos.udg.mx

\section{Juan Francisco Caldera Montes}

Profesor investigador de la Universidad de Guadalajara, México, con Perfil Deseable por parte del Programa para el Desarrollo del Profesorado (PRODEP), Doctor en Ciencias; forma parte del grupo de investigación en consolidación "Educación y Sociedad", actualmente cuenta con el reconocimiento como investigador Nivel 1 que otorga el 
M. R. Carranza Alcántar y J. F. Caldera Montes

Sistema Nacional de Investigadores del CONACYT. ORCID ID: 0000-0002-8999-3736.

Email: jfcaldera@cualtos.udg.mx 